\title{
Status of CONRAD, a nuclear reaction analysis tool
}

\author{
C. De Saint Jean a, B. Habert, O. Litaize, G. Noguere, and C. Suteau \\ CEA, French Atomic Energy Commission, Nuclear Energy Directorate, CEA/Cadarache, 13108 Saint-Paul-lez-Durance Cedex, France
}

\begin{abstract}
The development of a software tool (CONRAD) was initiated at CEA/Cadarache to give answers to various problems arising in the data analysis of nuclear reactions. This tool is then characterized by the handling of uncertainties from experimental values to covariance matrices for multi-group cross sections. An object oriented design was chosen allowing an easy interface with graphical tool for input/output data and being a natural framework for innovative nuclear models (Fission). The major achieved developments are a data model for describing channels, nuclear reactions, nuclear models and processes with interface to classical data formats, theoretical calculations for the resolved resonance range (Reich-Moore) and unresolved resonance range (Hauser-Feshbach, Gilbert-Cameron,...) with nuclear model parameters adjustment on experimental data sets and a Monte Carlo method based on conditional probabilities developed to calculate properly covariance matrices. The on-going developments deal with the experimental data description (covariance matrices) and the graphical user interface.
\end{abstract}

\section{Introduction}

The efforts made within our nuclear data group at CEA/Cadarache to give answers to various known limitations of existing software in the modelling of the physical phenomena and in the propagation of uncertainties to produce evaluations lead us to the development of a new software named CONRAD (for "COde for Nuclear Reaction Analysis and Dated Assimilation").

The objectives of this tool can be summarized as follows:

- development of a modern software tool for the analysis of nuclear processes allowing on-line treatment (input/output) via ergonomic user interfaces,

- become a powerful tool for evaluations in the field of resolved/unresolved resonances, incorporating a complete treatment of uncertainties from the experimental data to multi-group cross section used in reactor physics,

- allow the implementation of nuclear models more complex and predictive.

\section{CONRAD description}

The code is developed using $\mathrm{C}++$ programming language which allows the use of free numerical librairies largely widespread in the scientific field and ensure the perennial lifetime of the developments by the use of standards solutions of the public domain ("open source").

\subsection{General overview}

CONRAD is separated in four types of modules:

- general package

- theoretical package

- experimental package

- physical analysis package.

${ }^{a}$ Presenting author, e-mail: cyrille.de-saint-jean@cea.fr
The general package tries to glue all CONRAD modules in the same architecture with a dedicated data model. In this framework, data (experimental, physical or theoretical) are defined and interfaces with the formats of nuclear data files are developed. The theoretical package contains all "standard" models used in the determination of cross sections and provide a common base to simplify the writing and the coherence in the development of new models. The purpose of the experimental package is the development of everything that is related to the measurement of cross sections or other related nuclear quantities such as transmission, capture yields... As a results, resolution function, multiple scattering effects, operating condition description are coded in this frame. In addition, the sources of experimental uncertainties are treated rigorously with their associated covariance matrices. The aim of the physical analysis package is to provide a set of tools for the cross sections evaluation in the resolved and unresolved resonance range, with a proper interface with higher energy region. In particular, parameters estimations modules are developed in this frame. This package is, furthermore, the place where all uncertainties evaluations are grouped together to produce a final estimation of covariances on all model parameters, on multi-group cross sections taking into account all experimental errors (statistic and systematic).

\subsection{Architecture description}

CONRAD is developed in $\mathrm{C}++$ using object oriented programming (OO). This choice allows the simplification of interfaces (graphical interfaces with Matlab, Origin, Root, are simple to implement), makes the code independent of any data description by separating the internal data storage with formats (endf or others) and allows the use of an "easy" and "intuitive" description of the input data and what is necessary to describe a nuclear reaction (nuclear models with physical parameters and data, cross sections, collision matrices, list of openned channels ...). 


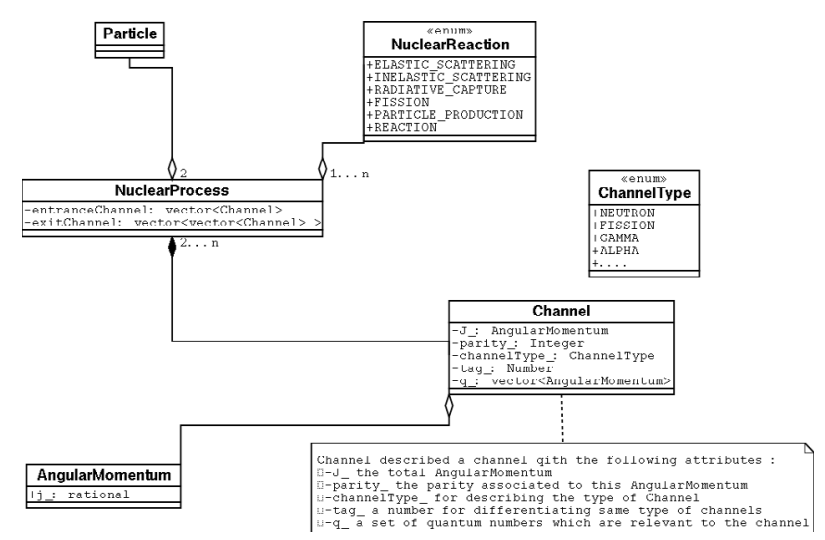

Fig. 1. UML data model description.

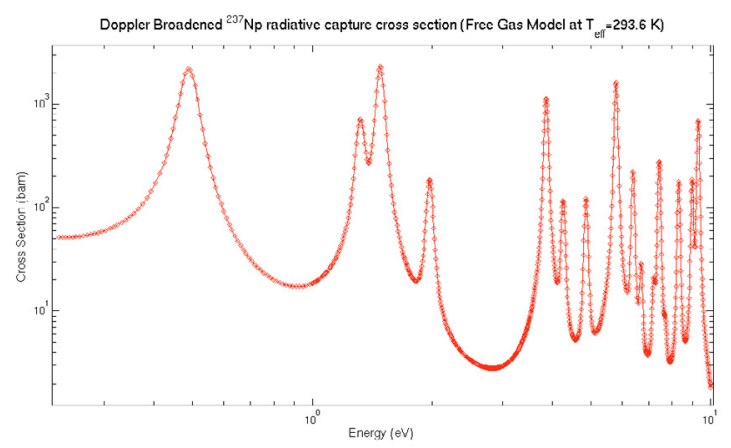

Fig. 2. ${ }^{237} \mathrm{~Np}$ capture cross section at room temperature.

\subsection{CONRAD data model}

The data model is presented in figure 1. It is used for describing channels, nuclear reactions and nuclear processes.

\section{Theoretical features}

All major nuclear models from the thermal range to the $\mathrm{MeV}$ range have been implemented.

In the resolved resonance range, both $\mathrm{R}$ Matrix approximation, Reich-Moore [1] and multi-level Breit-Wigner are coded using exactly the same kind of parameters.

In the unresolved resonance range, all needed recipies for average cross sections calculation [2-5] are coded such as Hauser-Feshbach theory with width fluctuations factors (Dresner, GOE triple integral), Moldauer's prescription, level density using Gilbert-Cameron formulaes, gamma transmission with Giant Dipole Resonance Model and Fission transmission with Hill-Wheeler multi-barrier description.

In addition, penetrability, phase shift and other kinematics variables usefull for both cross section calculations type are availiable.

Furthermore, in order to calculate cross sections at any temperature, a doppler broadenning tool is implemented with the possibility of choosing a free gaz model or a crystal lattice model ( $\mathrm{S} \alpha \beta$, phonon spectrum). An example of broadenned cross section in the resolved range is shown in figure 2.
Mathematical and numerical developments (integration, convolution, linear algebra...) are based on standard solutions coming from $\mathrm{C}++$ open-source librairies [10].

The architecture, relying on a generic data model, permits the development of new models based on a compound nucleus formation quite easily. The $\mathrm{C}++$ interface of these models must only give the transition probability between two channels of a corresponding nuclear process. All the cross sections counting $\left(\sigma_{c c^{\prime}}^{J \pi}, \sigma_{c}^{J \pi}, \sigma_{\text {total }}^{J \pi} \ldots\right)$ are then wrapped in modelindependent $\mathrm{C}++$ classes.

\section{Experimental features}

This module deals with all that is related to the treatment of experimental data sets and their uncertainties.

\subsection{Data reduction and experimental correlations}

This functionnality allows to look at raw uncorrelated data and produces the reduced long range correlated physical observables with all uncertainties taken into account (statistic and systematic).

This reduction operation consists of a succession of elementary operations to obtain the desired reduction function. The following elementary operations on data spectrum are implemented:

- $(\mathrm{a}+\delta \mathrm{a}) \cdot \mathrm{X}($ as well as division)

- X+ $\mathrm{X}^{\prime}, \mathrm{X} \cdot \mathrm{X}^{\prime}, \frac{X}{\mathrm{X}^{\prime}}$

- time to energy reconstruction,

- averaging and re-bining

- get isotope cross section from ENDF + some uncertainties (normalisation purpose).

Covariance matrices on resulting spectra are computed with the same theoretical method than in the code AGS [8]:

$$
M=S \cdot S^{T}+D
$$

$D$ is diagonal and represents the statistical part of the errors. $S$ is a reduced sized matrix taking into account the correlations coming from the data reduction process (normalization, background, dead time...).

Most of the data reduction functional can then be treated such as transmission, capture yields, fission...

An example is given for ${ }^{235} \mathrm{U}$ fission cross section measurements. The study is based on raw data measured at the IRMM by Serot et al. [12]. The figure 3 shows the good cross section reconstruction of the reduced experimental data with the CONRAD code by comparing with JEFF3.1 cross section. Figure 4 shows the corresponding experimental covariances.

\subsection{Multiple scattering module}

Multiple scattering is under development [7] using analytical solutions as well as monte-carlo simulations and treating cylinders (thin/thick samples) or spheres.

A comparison with the SAMMY code [9] was performed as shown in figure 5 on a gold sample. 


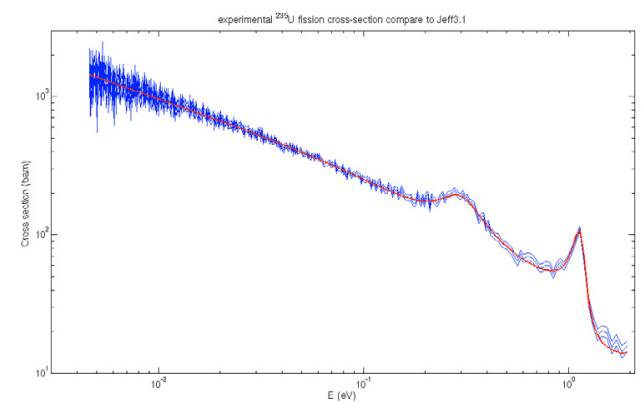

Fig. 3. $\sigma_{f}^{235} U$ obtained after reduction of the raw data measured at the IRMM (Serot et al. [12]) with CONRAD (red line is JEFF3.1).

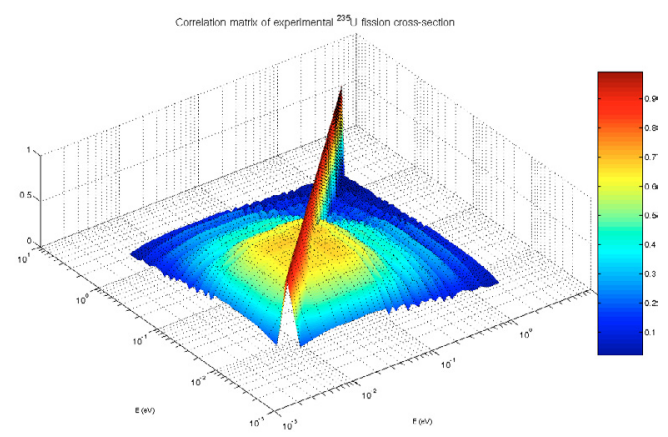

Fig. 4. ${ }^{235} \mathrm{U}$ fission measured cross section correlations.

\section{Analysis features}

CONRAD is designed to analyse nuclear data. This analysis can be, nuclear parameters estimation via adjustment on experiments, uncertainty propagation or statistical analysis of parameters. Thus, the following features are coded:

- a fitting wrapper based on Minuit2 coming from Root (Cern) framework [6] used in the resolved and unresolved resonance range to adjust classical model parameters $\left(\mathrm{E}_{\lambda}\right.$, $\left.\Gamma_{\lambda}^{c},<\Gamma^{c}>, \mathrm{S}_{l}, \mathrm{R}^{\infty} \ldots\right)$.

- a Monte-Carlo uncertainty propagation based on conditional probabilities was developed [11], this will be usefull for non-varied parameters uncertainty propagation

- some statistical analysis routines are coded for the study of resonance parameter distributions (level spacing, widths) and their comparaison with expected theoretical distributions (GOE predictions).

\subsection{Parameters estimation}

The fitting tool is interfaced with Minuit2 without any dependancies of CONRAD model parameters with the data type of this minimizer. The use of an alternative engine can then be introduced in the tool. A genuine mathematical package will thus be developed for a better description of any general lagrangian (Bayesian, least square...) chosen for minimization and a proper treatment of experimental covariances like equation (1).

The figure 6 shows an example of analysis of the average capture cross section of ${ }^{177} \mathrm{Hf}$ between $2 \mathrm{keV}$ and $100 \mathrm{keV}$.

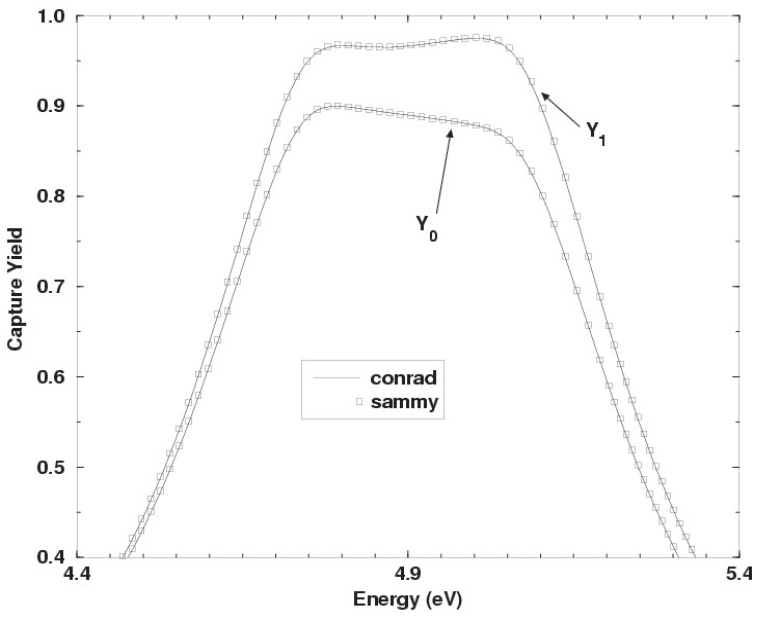

Fig. 5. Comparison between CONRAD and SAMMY for capture yields on a gold sample.

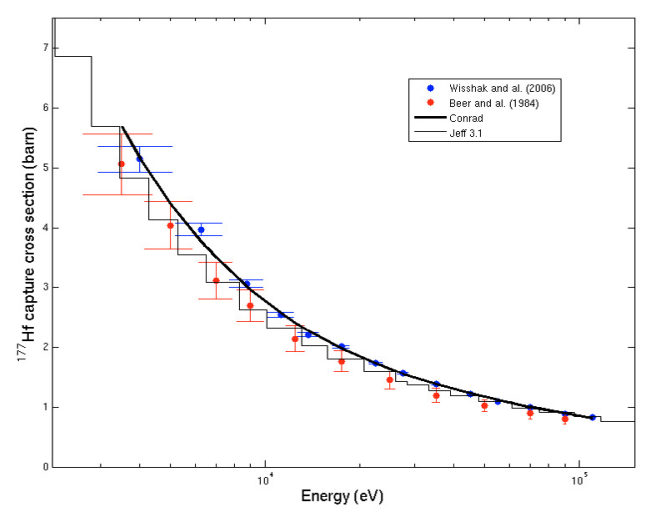

Fig. 6. Hauser-Feshbach analysis of the ${ }^{177} \mathrm{Hf}$ capture cross section. The starting neutron strength functions were obtained by using the DOM parameters established by V. Avrigeanu [13].

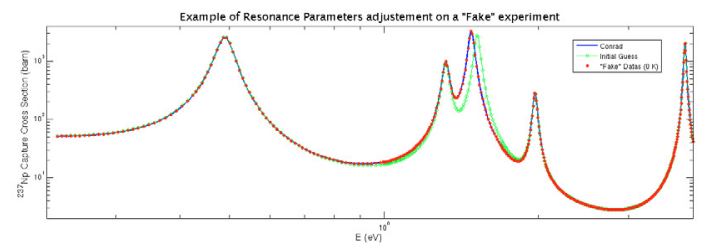

Fig. 7. Adjustement of the unbroadened ${ }^{237} \mathrm{~Np}$ capture cross section with the CONRAD code.

Here only two parameters were varied (average radiation widths). Other parameters were fixed to values coming from preliminary optical model calculations (for strength functions) or analysis of the resolved resonance range (level densities).

The figure 7 shows a "fake" analysis of ${ }^{237} \mathrm{~Np}$ capture cross section in the resolved resonance range. By fake, we mean that we have simulated an experiment at $T=0 \mathrm{~K}$ because for the time being only unbroadenned cross section can have their parameters adjusted. In this example, the position of the third resonance and the values of neutron widths of the first four resonances were slightly changed. 


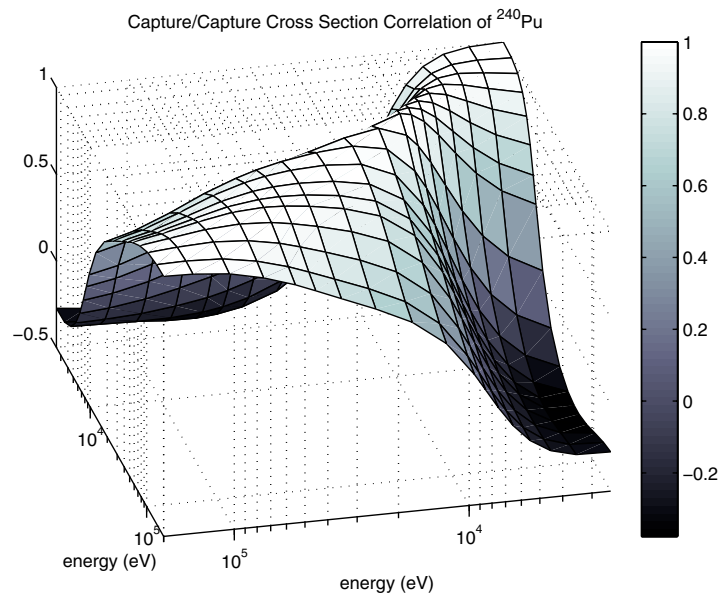

Fig. 8. ${ }^{240} \mathrm{Pu}$ capture/capture cross section correlations.

\subsection{Monte Carlo based uncertainty propagation}

A methodology previously exposed [11] to treat non-varying uncertain parameters and their influences on the estimation of other parameters with a fitting procedure is implemented in CONRAD with a simple interface to choose between adjusted or sampled (Monte Carlo) parameters. The final result of such a calculation (the cross section covariances) is presented in figure 8 for an academic ${ }^{240} \mathrm{Pu}$ total and capture evaluation.

\section{Conclusion}

The initial initiative of the nuclear data group of CEA/Cadarache has made CONRAD V1 to exist with mainly all objectives treated (uncertainties, simplified interface, room for models...). The tool does not pretend to have the same level of robustness of existing ones, but this promising first version will be used now on for evaluations and as a result will be tested and benchmarked by physicists.

The project is raising in term of man power, to fullfill the next main objective for 2008 which is the delivery of a graphical user interface allowing the fit of all known parameters from $0 \mathrm{eV}$ to $\mathrm{MeV}$ using any type of experiments, the interface with high energy code such as TALYS and/or ECIS for a coherent treatment of unresolved resonance range and an easy way of propagating uncertainties using dedicated calculation scheme.

The major on-going developments is then concerning fission models, resolution function implementation, a new minimizer engine and a graphical interface.

\section{References}

1. C.W. Reich, M.S. Moore, Phys. Rev. 111, 929 (1958).

2. W. Hauser, H. Feshbach, The Inelastic Scattering of Neutrons, Phys. Rev. Vol 87, No. 2 (1952).

3. P.A. Moldauer, Phys. Rev. C 14, 764 (1976).

4. J.J.M. Verbaarschot, H.A. Weidenmuller, M.R. Zirnbauer, Phys. Rep. 129, 367 (1985).

5. Gilbert, A.G.W. Cameron, Can. J. Phys. 43, 1446 (1965).

6. ROOT, an Object Oriented Data Analysis Framework, http://root.cern.ch.

7. O. Litaize et al. (these proceedings).

8. C. Bastian, P. Schillebeeckx et al., Proceedings of the PHYSOR2006 Topical Meeting on Reactor Physics, Vancouver, BC, Canada, 2006 (to be published).

9. N.M. Larson, Updated User's guide for SAMMY, OakRidge Report ORNL/TM-9179/R7 (2007).

10. Boost $\mathrm{C}++$ libraries, http://www.boost.org

11. C. De Saint Jean et al., Proceedings of the PHYSOR-2006 Topical Meeting on Reactor Physics, Vancouver, BC, Canada, 2006 (to be published).

12. O. Serot, C. Wagmans (pivate communication).

13. V. Avrigeanu et al., Nucl. Phys. A 765, 1 (2006). 